\title{
Filtering-Based Noise Estimation for Denoising the Image Degraded by Gaussian Noise
}

\author{
Tuan-Anh Nguyen and Min-Cheol Hong \\ Video and Processing Laboratory, Information and Telecommunication Department, \\ Soongsil University, 156-743 Sangdo-Dong, Dongjak-Gu, Seoul, Korea \\ \{anhnt, mhong\} assu.ac.kr
}

\begin{abstract}
In this paper, a denoising algorithm for the Gaussian noise image using filtering-based estimation is presented. To adaptively deal with variety of the amount of noise corruption, the algorithm initially estimates the noise density from the degraded image. The standard deviation of the noise is computed from the different images between the noisy input and its' pre-filtered version. In addition, the modified Gaussian noise removal filter based on the local statistics such as local weighted mean, local weighted activity and local maximum is flexibly used to control the degree of noise suppression. Experimental results show the superior performance of the proposed filter algorithm compared to the other standard algorithms in terms of both subjective and objective evaluations.
\end{abstract}

Keywords: Local statistics, Gaussian filtering, noise estimation, Denoising, Gaussian noise.

\section{Introduction}

Noise having Gaussian-like distribution is very often encountered in acquired data. Gaussian noise is characterized by adding to each image pixel a value from a zeromean Gaussian distribution. In the field of image processing and computer vision, noise removal while preserving image features such as edge, detail, and texture is a key problem [1].

In the past decades, there have been many attempts to construct digital filters which have the qualities of noise attenuation and detail preservation. For impulsive noise, the median filter and their modified approaches have been widely used due to their low computational cost benefits [1], [2], [3]. However, conventional noise reduction algorithms assume that the standard deviation of the Gaussian noise or noise level is known a priori, which is not valid in practical cases. Therefore, it is necessary to estimate the noise beforehand to apply these methods to some subsequent processes such as edge detection and object recognition for various applications.

In this paper, we propose a spatially adaptive denoising algorithm for image corrupted by Gaussian noise. Using local statistics to reflect a human visual system (HVS)[1], the noise level is obtained by filtering-based noise estimation, the noise detection function is defined to discriminate between true pixels and damaged one in the degraded image. In addition, under the assumption that an image is locally 
Gaussian-distributed with different activity, a modified Gaussian filter is defined to remove corrupted components, where the parameters of the Gaussian filter are also determined by local statistics.

The rest of the paper is organized as follows. Section 2 addresses the noise variance estimation approaches. Constrains for noise estimation, the proposed noise detection and modified Gaussian filtering based on the local statistics are described in Section 3. In sections 4 , the simulation results and performance comparisons will be presented to demonstrate the capability of the proposed algorithm. And finally section 5 reports conclusion.

\section{Noise Variance Estimation Approaches}

A common aim of image processing is to recover the original image $x$ from the degraded image $y$ that is contaminated by some noise model such as additive zeromean white Gaussian noise $n$.

$$
y=x+n \text {. }
$$

Knowing the amount of noise is very important to allow other algorithms adaptively filtering images instead of using fixed thresholds. Many algorithms have been proposed in this field and usually the exact value of the noise variance $\sigma_{n}^{2}$ is required as a crucial filter parameter. However, the main difficulty intermixing of the statistics of original image $x$ and the noise $n$ due to (1). The separation of the two signals is not an easy task and it is well known that the noise variance of the sum of two independent signals is the sum of the variances of the two components.

Generally, noise estimation algorithms in the spatial domain are classified into two approaches: block-based and filtering-based (smoothing-based) [4]-[6].

In block-based methods [5], [6], images are tessellated into a number of $M \times N$ blocks. Standard deviations of intensity are computed for all the blocks and sorted. The block with the smallest standard deviation has the least change of intensity. The smaller the standard deviation is, the smoother the block. The intensity variation of a smooth block may be due to noise, in which the standard deviation of the block is close to that of the Gaussian noise added. The smallest standard deviation of a block of intensity is assumed to be equal to that of the additive white Gaussian noise. This algorithm is simple, but tends to overestimate the amount on noise for small noise cases. Noise can be underestimated in large noise cases. Also, the main difficulty of these methods is that their estimates may vary significantly depending on the input image and noise levels.

In filtering-based methods [4], a noisy input image is filtered by a low-pass filter to suppress the image structure. The standard deviation $\hat{\sigma}_{n}$ of the different image between the noisy input image and its filtered image is computed as illustrated in Fig. 1. This method especially yields good estimates for large noise cases [7]. 


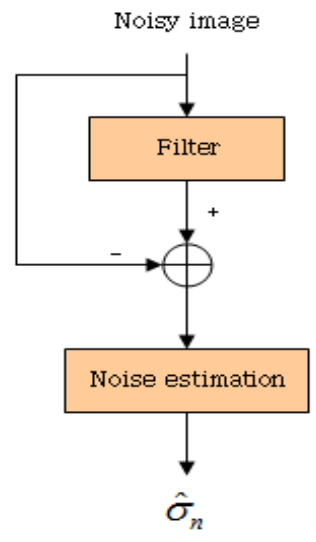

Fig. 1. Filtering-based noise estimation

\section{Proposed Denoising Algorithm}

The typical image degradation model at point $(i, j)$ in a two dimensional coordinate is written as in (1). The problem at hand is to know exactly noise level on image in order to incorporate the most appropriate filter method. In this paper, a noise estimation algorithm bases on filtering using the local statistics is proposed. It is non-recursive and does not require the use of any kind of transforms. It shares the same characteristics in that each pixel is processed independently. Consequently, this approach has an obvious advantage when used in real-time digital image processing applications.

In addition, the local weighted mean, local weighted variance and local weighted max play an significant role to the denoising stage since its' advantages [8], [9], we use them as the key factors to establish the Modified Gaussian filter. For a pixel of the observed image $y$, the local weighted mean and the local weighted activity with the window of size $(2 U+1) \times(2 V+1)$ are defined as $(2)$.

$\mu_{i, j}=\frac{\sum_{m} \sum_{n,(m, n) \in S_{1}} w_{m, n} \hat{x}_{i+m, j+n}+\sum_{m} \sum_{n,(m, n) \in S_{2}} w_{m, n} y_{i+m, j+n}}{\sum_{m=-U}^{U} \sum_{n=-V}^{V} w_{m, n}}$,

$\sigma_{i, j}=\frac{\sum_{m} \sum_{n,(m, n) \in S_{1}} w_{m, n}\left|\hat{x}_{i+m, j+n}-\mu_{i, j}\right|+\sum_{m} \sum_{n,(m, n) \in S_{2}} w_{m, n}\left|y_{i+m, j+n}-\mu_{i, j}\right|}{\sum_{m=-U}^{U} \sum_{n=-V}^{V} w_{m, n}}$

In (2), $w_{m, n}$ denotes the weighting coefficient at the point $(m, n)$ within the window, and $|\bullet|$ represents the absolute operator. Also, $\hat{x}_{i+m, j+n}$ denotes the value of the reconstructed pixel at the point $(i+m, j+n)$ belonging to $S_{1}$, where $S_{1}$ and $S_{2}$ represent the causal region (dark region) and the non-causal region (white region) with respect to the point $(i, j)$ in progressive scanning order, and the intersection between $S_{1}$ and $S_{2}$ is null, as shown in Fig. 2. 


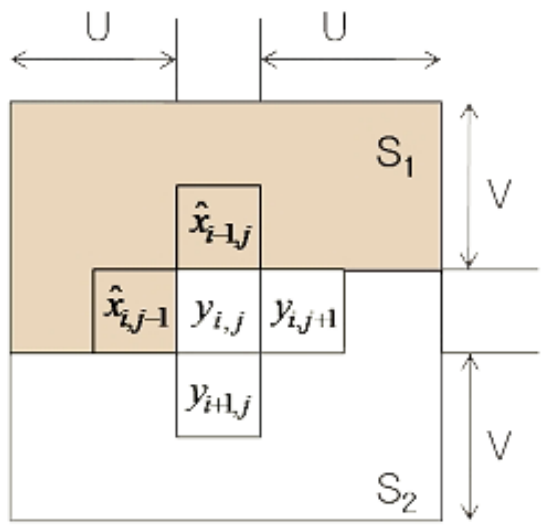

Fig. 2. Local window for determining weighted local information

Also, the local maximum of the observed image is

$$
y_{\max , i, j}=\max _{(p, q) \in S} y_{p, q} .
$$

where $S$ is the support region to determine the local maximum about the point $(i, j)$. In this work, $S$ is the same as the analysis window used for local weighted mean and local weighted activity $\left(S_{1} \cup S_{2}=S\right)$. In order to effectively obtain the better pre-filtered image, we define the noise detection stage, which can discriminate noisy and noise-free pixels prior to applying the noise removal filter. Under the assumption that an image is locally Gaussian-distributed with local smoothing constraint, a noise detection function as in (4)

$$
\begin{aligned}
& \text { flag }_{i, j}=\left\{\begin{array}{c}
1 \quad \text { if } y_{i, j}>\mu_{i, j}+B_{i, j} \\
\quad \text { or } y_{i, j}<\mu_{i, j}-B_{i, j} . \\
0 \text { otherwise }
\end{array}\right. \\
& B_{i, j}=k \times \frac{\sigma_{i, j}}{y_{\max , i, j}},
\end{aligned}
$$

In (4), $k$ represents a constant. This equation means that the value of the flag is equal to 1 when a pixel is detected as a corrupted one. It is clear how the local statistics affects the detection function. Assume that a same noise is added to both the flat area and the high activity region with the same local maximum. In this case, $B_{i, j}$ of the flat area is relatively smaller than that of the high activity region. The smaller $B_{i, j}$ represents tighter bounds for the flat region, so that small variations of the flat can be detected as corrupted pixels. On the other hand, a higher activity region leads 
to looser bounds and small variations of the high activity area are not detected as uncorrupted pixels, leading to the preservation of important features. This is in agreement with the noise masking property of the HVS [1].

According to the above condition, when a pixel is detected as the corrupted one with lower local weighted activity, strong filtering (over-smoothing) process is required, while weak filtering for the corrupted pixels with higher activity. Gaussian filter is very useful to control the degree of the smoothness of the reconstructed image by using the local activity.

Using the local statistics in (2), we propose a modified Gaussian filter such as

$$
h_{i, j}=\frac{1}{Z_{i, j}} \exp \left(-T \frac{\sigma_{i, j}^{2}\left(i^{2}+j^{2}\right)}{\sqrt{\mu_{i, j}+1}}\right) .
$$

where $Z_{i, j}$ and $T$ denote the normalizing constant and a tuning parameter, respectively. Also, the support region of the Gaussian filter in (5) is the same as that of the analysis window in (3). Then, the reconstructed pixel can be written as (6). For the corrupted pixels with small local activity (flat region), (6) leads to strong filtering since the filter coefficients within the support region have similar values. On the other hand, it results in weak filtering for the corrupted pixels with large local activity.

$$
\hat{x}_{i, j}= \begin{cases}\frac{\sum_{m} \sum_{n,(m, n) \in S_{1}} h_{m, n} \hat{x}_{i+m, j+n}+\sum_{m} \sum_{n,(m, n) \in S_{2}} h_{m, n} y_{i+m, j+n}}{\sum_{m=-U}^{U} \sum_{n=-V}^{V} h_{m, n}} & \text { if } \text { flag }_{i, j}=1 \\ y_{i, j} & \text { otherwise }\end{cases}
$$

To incorporate the most appropriate filtering method, the noise variance levels on the input degraded image are estimated as in Fig. 3, in which as the estimated noise less than the value alpha, the weighting coefficients are the diagonal element within the $(2 U+1) \times(2 V+1)$ support window to avoid the over-smoothness problem. In this case, it means that the noise level is relatively low (greater than $20 \mathrm{~dB}$ and $30 \mathrm{~dB}$ ). Hence, it is better to use only 5 pixels in the cross region of the $3 \times 3$ support window to calculate the local weighted mean $\mu_{i, j}$, local weighted variance $\sigma_{i, j}$ and the output pixel $\hat{x}_{i, j}$. On the other hand, as the estimated noise greater than the alpha, the input image is corrupted seriously (less than $10 \mathrm{~dB}$ ). It is, therefore, necessary to incorporate more pixels for the processing. The uniform weighting coefficients and all pixels within the $(2 U+1) \times(2 V+1)$ support window are used to calculate the local information.

The novelty of the proposed algorithm is that it has the capability to effectively remove the noise components using the local statistics and estimate the image's noise density for flexibly treats different amount of corruptions. 


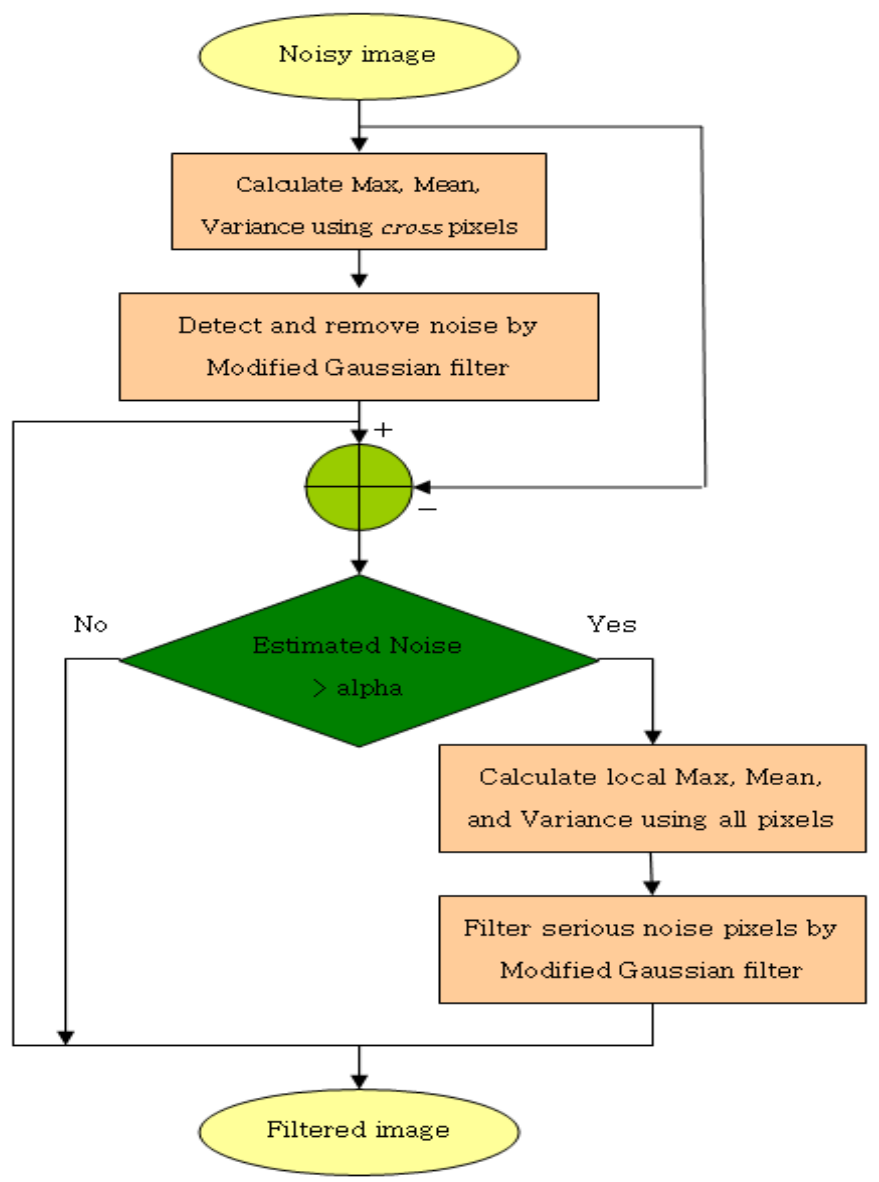

Fig. 3. Entire procedure of proposed algorithm

\section{Experimental Results and Performance Comparisons}

The proposed algorithm was tested with various Gaussian noise images for various SNRs such as "Lena", "Cameraman", "Bird", "Goldhill" images and compared them with pixelwise median absolute difference (PWMAD)[10], rank-order criterion filter (ROC)[11], switching-based adaptive weighted mean (SAWM)[12] and bilateral filter (BF)[13].

In order to evaluate the performance of the noise detection algorithm, the following noise detection fidelity $\left(D_{F}\right)$ was used. It is

$$
D_{F}=\left(1-\frac{F_{P}+M_{P}}{T_{P}}\right) \times 100 .
$$


In (7), $F_{P}, M_{P}$, and $T_{P}$ represent the number of detection "fault" pixels, the number of detection "missing" pixels, and the number of total pixels in an image, where "fault" means that an uncorrupted pixel is detected as a corrupted one. On the other hand, "missing" denotes that a corrupted pixel is considered as an uncorrupted one. In our work, alpha $=4, U=1, V=1$ and the uniform weighting coefficients in (2) are used. For evaluating the performance of noise filtering, we use peak signal-tonoise ratio (PSNR) and universal image quality index (UIQI) which is a new method for image assessment [14].

Table 1. Performance comparisons of Lena image

\begin{tabular}{ccccc}
\hline Noise & Method & $D_{F}$ & PSNR & UIQI \\
\hline \multirow{4}{*}{$10 \mathrm{~dB}$} & PWMAD & 82.04 & 27.77 & 0.639 \\
& ROC & 81.91 & 27.61 & 0.635 \\
& SAWM & 84.96 & 27.84 & 0.632 \\
& BF & N/A & 29.43 & 0.698 \\
& Proposed & 94.98 & 29.45 & 0.718 \\
\hline \multirow{4}{*}{$20 \mathrm{~dB}$} & PWMAD & 71.83 & 30.64 & 0.797 \\
& ROC & 70.43 & 30.18 & 0.796 \\
& SAWM & 84.86 & 30.95 & 0.800 \\
& BF & N/A & 32.28 & 0.830 \\
& Proposed & 89.61 & 32.36 & 0.851 \\
\hline \multirow{4}{*}{$30 \mathrm{~dB}$} & PWMAD & 67.37 & 31.24 & 0.852 \\
& ROC & 55.84 & 31.66 & 0.854 \\
& SAWM & 70.03 & 31.9 & 0.857 \\
& BF & N/A & 32.63 & 0.860 \\
& Proposed & 70.03 & 33.09 & 0.914 \\
\hline
\end{tabular}

Table 2. Performance comparisons of Cameraman image

\begin{tabular}{ccccc}
\hline Noise & Method & $D_{F}$ & PSNR & UIQI \\
\hline \multirow{4}{*}{$10 \mathrm{~dB}$} & PWMAD & 83.94 & 25.08 & 0.369 \\
& ROC & 82.67 & 25.06 & 0.368 \\
& SAWM & 90.14 & 25.93 & 0.389 \\
& BF & N/A & 27.93 & 0.441 \\
& Proposed & 95.65 & 28.11 & 0.445 \\
\hline \multirow{5}{*}{$20 \mathrm{~dB}$} & PWMAD & 83.85 & 27.06 & 0.516 \\
& ROC & 78.65 & 27.34 & 0.515 \\
& SAWM & 86.02 & 27.38 & 0.522 \\
& BF & N/A & 31.22 & 0583 \\
& Proposed & 93.62 & 29.77 & 0.612 \\
\hline \multirow{5}{*}{$30 \mathrm{~dB}$} & PWMAD & 70.32 & 27.32 & 0.644 \\
& ROC & 69.33 & 27.76 & 0.656 \\
& SAWM & 70.95 & 27.7 & 0.649 \\
& BF & N/A & 31.62 & 0.678 \\
& Proposed & 79.04 & 30.31 & 0.784 \\
\hline
\end{tabular}




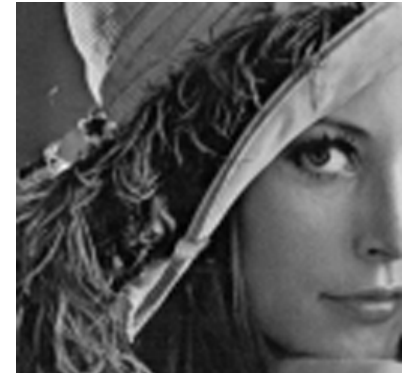

(a)

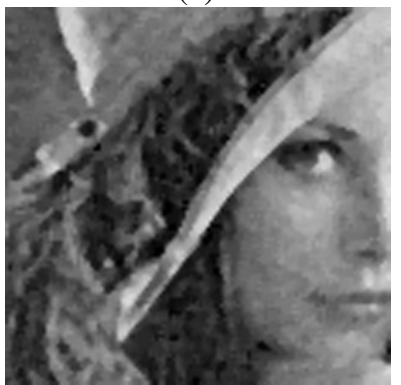

(c)

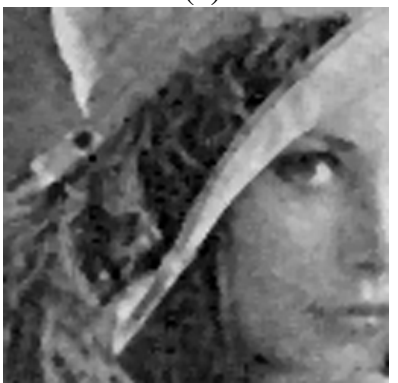

(e)

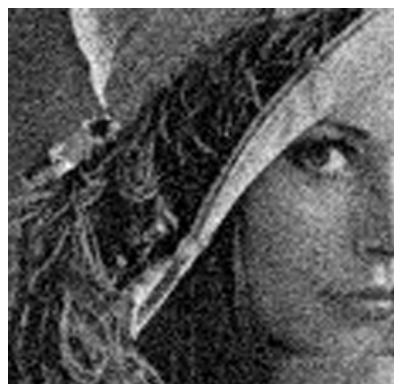

(b)

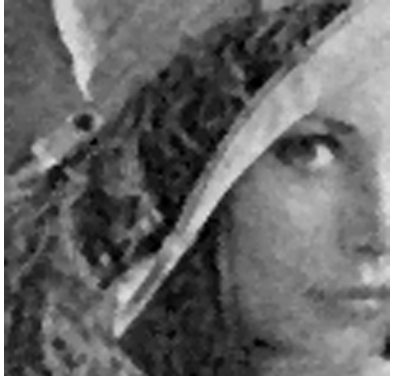

(d)

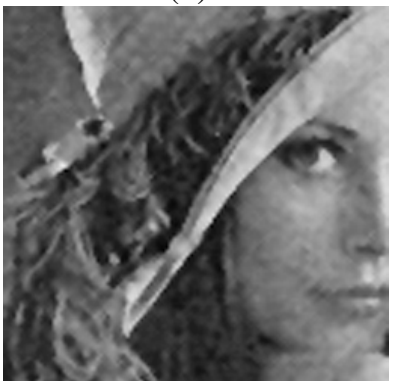

(f)

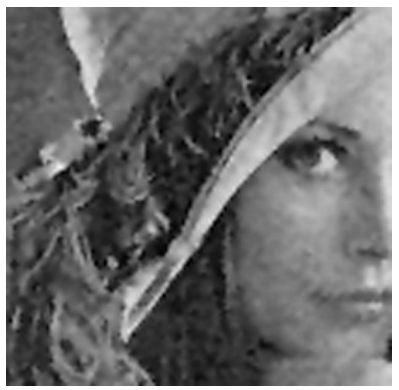

(g)

Fig. 4. Experimental results of Lena image: (a) enlarged original image, (b) enlarged degraded image with $10 \mathrm{~dB}$ Gaussian noise, (c) corresponding reconstructed image with PWMAD, (d) corresponding reconstructed image with ROC, (e) corresponding reconstructed image with SAWM, (f) corresponding reconstructed image with $\mathrm{BF}$, (g) corresponding reconstructed image with proposed algorithm 


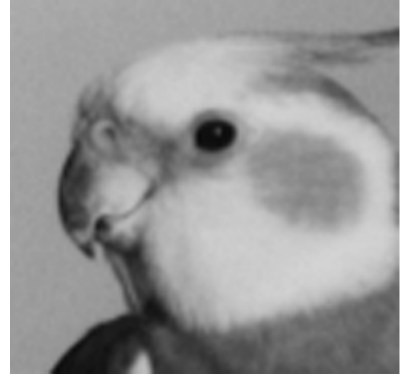

(a)

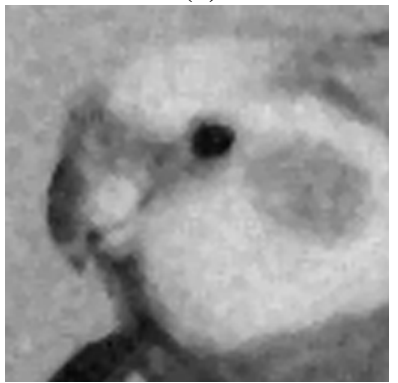

(c)

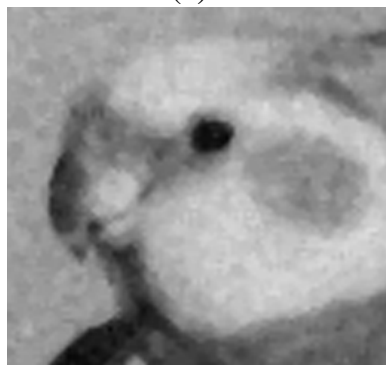

(e)

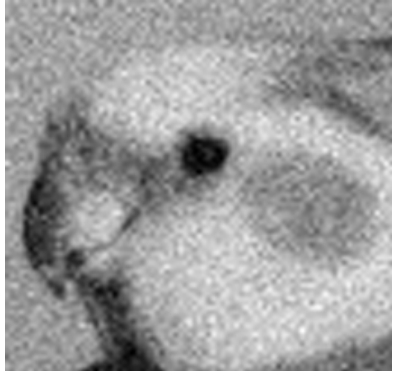

(b)

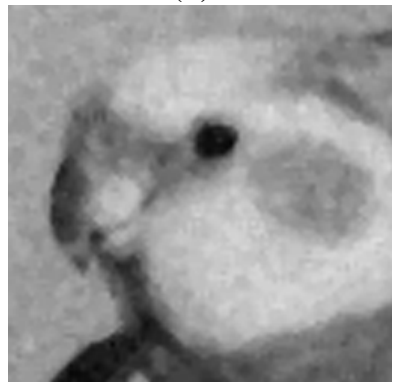

(d)

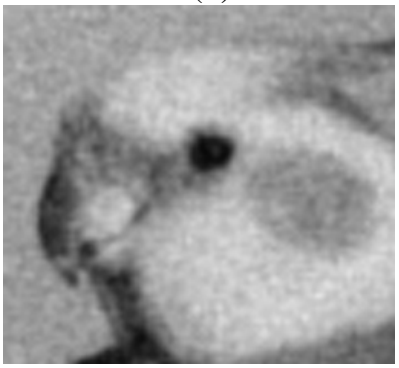

(f)

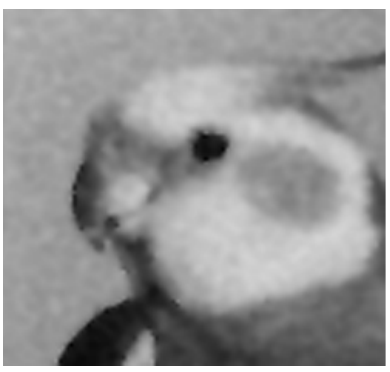

(g)

Fig. 5. Experimental results of Bird image: (a) enlarged original image, (b) enlarged degraded image with $10 \mathrm{~dB}$ Gaussian noise, (c) corresponding reconstructed image with PWMAD, (d) corresponding reconstructed image with ROC, (e) corresponding reconstructed image with SAWM, (f) corresponding reconstructed image with BF, (g) corresponding reconstructed image with proposed algorithm 
As shown in Fig. 4 and Fig. 5, PWMAD, ROC, SAWM lead to over-blurred reconstructed results. We observed that the degree of the smoothness is more serious as the additive noise is smaller since the degree of the additive noise is not considered in the filtering process of the methods. On the other hand, BF and proposed algorithm lead to the effective noise removal with preservation of the important features.

Table 1 and Table 2 summarize the performance comparisons with respect to $D_{F}$, PSNR and UIQI. They show that $D_{F}$ of ours outperforms the other approaches for all cases (BF does not require noise detection procedure). In addition, it was observed that $\mathrm{BF}$ and proposed algorithm are the most competitive among the above approaches in terms of PSNR and UIQI. However, the gain is getting smaller as the images are seriously contaminated.

\section{Conclusions}

In this paper, a denoising algorithm for the Gaussian noise image using filtering-based estimation is presented. This shows an efficient noise denoising algorithm that takes into account the filtering-based noise estimation algorithm and noise detection that it leads to objectively and subjectively satisfactory results without prior information about the noise by incorporating the local statistics. Currently, we are improving the noise estimate stage to obtain a more sophisticated formulation can be derived and better performance.

Acknowledgments. This work was supported by the Korea Science and Engineering Foundation (KOSEF) grant fund by the Korea Government (MEST) (No. 20110000148) and the Ministry of Knowledge Economy, Korea under the Information Technology Research Center support program supervised by the National IT Industry Promotion Agency [NIPA-2011-(C1090-1111-0003)].

\section{References}

1. Arce, G.R.: Nonlinear signal processing: A Statistical approach. John Wiley and Sons Inc. (2004)

2. Nodes, T.A., Gallagher, N.C.: Median filters: some modifications and their properties. IEEE Trans. Acoustics, Speech and Signal process. 30(5), 739-746 (1982)

3. Bednar, J.B., Watt, T.K.: Alpha-trimmed means and their relationship to median filter. IEEE Trans. Acoustics, Speech and Signal Process. 32(1), 145-153 (1984)

4. Olsen, S.I.: Noise Variance Estimation in Images: An evaluation, Computer Vision Graphics Image Processing. Graphic Models and Image Processing 55(4), 319-323 (1993)

5. Lee, J.S., Hoppel, K.: Noise modeling and estimation of remotely-sensed image. In: International Conference on Geoscience and Remote Sensing, Vancouver, Canada, vol. 2, pp. 1005-1008 (1989)

6. Shin, D.H., Park, R.H., Yang, S.J.: Block-based noise estimation using adaptive Gaussian filtering. IEEE Trans. on Consumer Electronics 51(1) (2005)

7. Rank, K., Lendl, M., Unbehauen, R.: Estimation of image noise variance. IEEE Proc. Vision Image Signal Process. 146, 8-84 (1999) 
8. Lee, J.S.: Refined filtering of image noise using local statistics. Computer Vision, Graphics and Image processing 15, 380-389 (1989)

9. Mastin, G.A.: Adaptive filters for Digital noise smoothing, An evaluation. Computer vision, Graphics and Image processing 31, 103-121 (1985)

10. Crnojevic, V., Senk, V., Trpovski, Z.: Advanced impulse detection based on pixel-wise MAD. IEEE Signal Process. Letters 11(7), 589-592 (2004)

11. Aizenberg, I., Butakoff, C.: Effective impulse detector based on rank-order criteria. IEEE Signal Process. Letters 11(3), 363-366 (2004)

12. Zhang, X., Xiong, Y.: Impulse noise removal using directional differences based noise detector and adaptive weighted mean filter. IEEE Signal Process. Letters 16(4), 295-298 (2009)

13. Elad, M.: On the origin of the bilateral filter and ways to improve it. IEEE Trans. Image Process. 11(10), 1141-1151 (2002)

14. Wang, Z., Bovik, A.C.: A universal image quality index. IEEE Signal Processing Letters 9(3), 81-84 (2002) 